KATARZYNA LUKAS

\title{
Mythos, Archetyp und Translation. Die Prosa von Bruno Schulz im Kontext der Ideen von Thomas Mann und Carl Gustav Jung
}

Artykuł skupia się na relacjach między pisarstwem Brunona Schulza (1892-1942) a refleksją filozoficzno-kulturową Tomasza Manna oraz psychologią C.G. Junga. Twórczość Schulza ujmowana jest jako przekład interdyskursywny takich koncepcji, jak: mit, archetyp i nieświadomość zbiorowa, które autor Mityzacji rzeczywistości przekształca we własny program poetologiczny. Dostępne dziś przekłady na język niemiecki nie odzwierciedlają tej relacji intertekstualnej: nie przekazują kluczowej idei artysty jako medium nieświadomości zbiorowej, implikują ponadto filozofię języka odmienną od tej, która zawarta jest w twórczości Schulza.

Der Beitrag handelt von den Beziehungen zwischen den Werken des polnisches Schriftstellers Bruno Schulz (1892-1942) und der kulturphilosophischen Reflexion von Thomas Mann sowie der Tiefenpsychologie von C.G. Jung. Das Euvre von Schulz erscheint als diskursive Translation von Konzepten wie dem Mythos, dem Archetyp und dem kollektiv Unbewussten, die der polnische Autor in sein eigenes poetologisches Programm überträgt. Die vorhandenen deutschen Übersetzungen von Schulz' Texten geben diese intertextuelle Relation nicht wieder: Sie sind nicht in der Lage, die Schlüsselidee vom Künstler als Medium des kollektiv Unbewussten zu vermitteln und implizieren eine andere Sprachphilosophie als diejenige, die im Werk von Schulz verschlüsselt ist.

The paper deals with the relations between the works of the Polish author Bruno Schulz (1892-1942), some anthropological and philosophical concepts of Thomas Mann and the depth psychology of C.G. Jung. Schulz's Euvre appears as an interdiscursive translation of such concepts as the mythos, the archetypes and the common unconscious - categories which Schulz transformed into his own poetical programme. The translations of Schulz' works into German do not render this intertextual relationship though. They do not convey the crucial idea of the artist 
Katarzyna Lukas

being a medium of the common unconscious, and imply a language philosophy which is different from that intended by the Polish writer.

\section{Translation: Ein übergreifender Zentralbegriff der Literatur- wissenschaft}

An der Schnittstelle zwischen Literaturwissenschaft und Übersetzungsforschung machen sich heutzutage zwei Tendenzen bemerkbar: erstens, die Translationswissenschaft mit Komparatistik zu identifizieren, und zweitens beide in der Kulturwissenschaft aufgehen zu lassen. Dies resultiert aus der Erschließung und Beanspruchung des Translationsbegriffes durch mehrere geisteswissenschaftliche Disziplinen - auch solche, die sich mit dem Phänomen der Übersetzung bisher kaum beschäftigt haben (BACHMANN-MEDICK 2009:260-272). Die Translation ist zurzeit allmählich zum Angelpunkt für Ethnologen, Anthropologen, Kultur- und Literaturwissenschaftler geworden, wobei sie sich nicht nur auf die herkömmliche linguistische Übersetzung beschränkt, sondern auch anthropologische, kulturelle, philosophische u.ä. Übertragungsprozesse umfasst. ${ }^{1}$ Die translatorische Orientierung der einzelnen Wissenschaften lässt sich insbesondere an den Folgen des ,cultural turn“ und des ,translational turn' mit aller Deutlichkeit beobachten. Die kulturelle Wende, angeregt durch Erkenntnisse der ethnologischen und anthropologischen Feldforschung (vorwiegend im angelsächsischen Raum), resultierte aus der Betrachtung fremder Kulturphänomene, sprachlicher und literarischer Erzeugnisse als Texte, die in die vertrauten Kulturzusammenhänge des Forschers grundsätzlich übertragbar seien. Die Übersetzungswissenschaft wurde damit mit Kulturwissenschaft und Kultursemiotik in Verbindung gebracht und von solchen Arbeitsbegriffen wie ,(Kultur)Text ${ }^{\star}$ (verstanden als statische, in sich geschlossene Entität) und ,interkultureller Transfer' dominiert. Demgegenüber geht die Kulturwissenschaft nach dem ,translational turn“ davon aus, Kultur selbst sei mit Übersetzung identifizierbar, indem sie durch Translationsprozesse hervorgebracht werde (BACHMANN-MEDICK 2009:245250). Akteure, die an diesen Vorgängen beteiligt sind, werden dynamisch

1 Daher wird zuweilen zwischen Übersetzung ,sensu stricto" und Translation differenziert: Die erste Kategorie umfasse vor allem linguistische, semantische und pragmatische Aspekte des Translationsvorgangs, während Translation sich auf anthropologische, ethnische, kulturelle, philosophische u.a. Prozesse erstrecke (DE TORO 2003:28). In diesem Beitrag werden die Begriffe der Einfachheit halber synonym verwendet. 
aufgefasst: als Größen, die sich gegenseitig beeinflussen, ihre Gestalt verändern und eine hybride Identität entwickeln können. Dabei wird das jeweils ,Fremde“ - die fremde Sprache, fremde Sitten, Weltbilder, literarische Werke - nicht erschlossen oder ,domestiziert', sondern in seiner Fremdheit bewahrt und akzeptiert. Aus den beiden Paradigmenwechseln, deren methodologische Resultate sich übrigens nicht ausschließen, sondern gegenseitig ergänzen, ergeben sich nun neue Grundeinsichten und Forschungspostulate für die Geisteswissenschaften im Allgemeinen und die Translationswissenschaft im Besonderen.

Was sich die Übersetzungsforschung nach dem ,translational turn` zum Ziel setzt, ist weniger die Bewertung der ,Treue' des Zieltextes zum Original als die Verfolgung der Strategien zur Konstruktion des Fremden in der Übersetzung (BACHMANN-MEDICK 2004a:159). Es werden zudem Identitäten, Räume, Kulturphänomene untersucht, denen das Merkmal Hybridität anhaftet - semiotische „Mischprodukte“, die infolge von Wechselbeziehungen in Randgebieten zwischen verschiedenen Kulturen (in „Übersetzungsräumen“) entstehen (BACHMANN-MEDICK 2004a:285-287). Für die Geisteswissenschaften bestehen die Konsequenzen der beiden Wenden in der Betonung der Transdisziplinarität und des Translationsbegriffes. Die Paradigmen, , alles ist Text' sowie , alles ist Übersetzung', fördern den transdisziplinären Charakter der Literaturwissenschaft, die auf die benachbarten Wissensgebiete übergreift. Sie verlangen aber auch, den Begriff ,Übersetzung' möglichst weit abzustecken. Der letztere ist auf Grund seiner Flexibilität und Umfunktionierbarkeit besonders gut geeignet, als gemeinsamer Nenner für Soziologie, Ethnologie, Psychologie, Linguistik, Literaturwissenschaft usw. zu dienen. Die Zentrierung um die Übersetzungskategorie bedeutet, dass jeder nach ihr ausgerichtete Wissenschaftsbereich zunehmend mit der Translationswissenschaft fusioniert, da der Übersetzung praktisch jede Form intertextueller Beziehung unterfallen kann.

Daher erscheint es legitim, die Komparatistik nach BRZOSTOWSKA-TERESZKIEWICZ (2004:320f.) neu zu definieren, indem man den Forschungsgegenstand der Disziplin auf Roman Jakobsons altbewährte Dreiteilung von Übersetzungsphänomenen zurückführt. Die Vergleichende Literaturwissenschaft beschäftige sich demnach mit der Übersetzung, sensu largo': neben der herkömmlichen interlingualen (rein sprachlichen) Übersetzung auch mit der intersemiotischen Translation (Beziehungen zwischen Literatur und anderen Künsten) und der intralingualen Translation, unter welcher die Übertragung von Diskursen zu verstehen wäre. Die Identifizierung der Translationswissen- 
schaft mit der Komparatistik bietet der letzteren die Chance, ihren Gegenstand umzudenken und neue Perspektiven zu erschließen. Die umorientierte Vergleichende Literaturwissenschaft schließt sich nun der Kulturforschung an, indem sie die Vielfalt von translatorischen, translationsähnlichen und -verwandten Erscheinungsformen betont, die in der Literatur nicht isoliert, sondern als Bündel von Kulturphänomenen auftreten. Zur Verschränkung von verschiedenen Translationstypen kommt es, wenn ein Schriftsteller beispielsweise ein fremdes, in einer Fremdsprache festgehaltenes Gedankengut dem eigenen Schaffen einverleibt und in seiner Muttersprache künstlerisch verarbeitet. Dies ist der Fall, wenn der polnisch-jüdische Autor Bruno Schulz (1892-1942) sich mit psychoanalytischen Erkenntnissen von C.G. Jung und der anthropologischen Reflexion von Thomas Mann auseinandersetzt. Dieses intertextuelle Gefüge, das sich wohl am ehesten als interdiskursive Translation bezeichnen ließe, soll im ersten Teil dieses Beitrags behandelt werden. In einem weiteren Teil liegt der Schwerpunkt auf der interlingualen Übersetzung, d.h. den Übertragungen der Schriften von Bruno Schulz ins Deutsche.

\section{Bruno Schulz: eine hybride Identität, ein hybrider Kulturtext}

Der heutigen Komparatistik und Translationswissenschaft, die sich vornehmlich hybriden literarischen Phänomenen und Kulturtexten zuwenden, bieten sich sowohl das Werk als auch die Künstlerpersönlichkeit von Bruno Schulz als geeigneter Untersuchungsgegenstand an. Der Polnisch schreibende Schriftsteller, Literaturkritiker, Maler und Graphiker, mit seiner Heimatstadt Drohobycz in Galizien - im nordöstlichen Grenzgebiet des ehemaligen Habsburgerreiches - lebenslang verbunden, war gleichermaßen in der polnischen, jüdischen und deutschen Kultur beheimatet. Obwohl zweisprachig und mit einem multikulturellen Hintergrund, ohne den sein dichterisches und zeichnerisches Euvre undenkbar gewesen wäre, vermittelt er darin kein Bild einer konkreten Nationalkultur, sondern vielmehr die geistige Atmosphäre eines verschlafenen Provinzstädtchens der Donaumonarchie um die Jahrhundertwende. Unter seinen literarischen Wahlverwandtschaften stehen diejenigen mit der deutschsprachigen Literatur im Vordergrund: mit Thomas Mann, Franz Kafka, Robert Musil, Joseph Roth, Alfred Kubin u. a. Allerdings sind konkrete stilistische und motivische Ähnlichkeiten bzw. Inspirationen spärlich 
und schwer aufzuzeigen ${ }^{2}$ - eines von mehreren Paradoxen im Schaffen des polnischen Autors. Aus diesem Grund kann man in Bruno Schulz eine hybride Dichterindividualität par excellence sehen. Sein Werk, das vor allem aus den beiden Erzählbänden Sklepy cynamonowe (Die Zimtläden) und Sanatorium pod Klepsydra (Das Sanatorium zur Todesanzeige) besteht, ist ein Gefüge von literarischen Motiven, philosophischen Gehalten und stilistischen Merkmalen unterschiedlicher Herkunft, es lässt sich allerdings nicht in einzelne Bausteine zerlegen. Auch die ethnische, religiöse und sprachliche Identität von Bruno Schulz ist in einem „hybriden Übersetzungsraum“ angesiedelt. ${ }^{3}$ Im Laufe der Zeit werden dem Verfasser der Zimtläden auf Grund seiner Persönlichkeit und seiner tragischen Biographie (er wurde von einem SS-Offizier auf offener Straße erschossen) symbolhafte Züge zugewiesen: Er wird heute als Sinnbild eines dem Holocaust zum Opfer gefallenen genialen Künstlers rezipiert. Das Zeichenhafte seines Schaffens und seiner Person beweisen nicht nur wissenschaftliche Untersuchungen, sondern auch z.B. literarische Werke von Gegenwartsautoren, die an den Lebenslauf von Schulz anknüpfen oder ihn frei verarbeiten. ${ }^{4}$ Der Dichter als Kulturtext: so ließe sich die Position von Bruno Schulz (verstanden als Mensch und als Werk) im heutigen literarischen, literatur- und kulturwissenschaftlichen und nicht zuletzt dem translationswissenschaftlichen Diskurs festhalten.

\section{3. Übertragung von Diskursen: der Mythos und das kollektive Unbewusste}

Neben der Hybridität seines Werkes und seiner Dichterpersönlichkeit ist Bruno Schulz deswegen für die Komparatistik von Interesse, weil sich in seinem Euvre die Übertragung von verschiedenen Diskursen in eine eigene Poetik verfolgen lässt. Es handelt sich dabei nicht nur um die literarische Intertextualität (die sich z.B. im Motiv Galiziens niederschlägt), sondern in

2 Am häufigsten wird wohl auf das Motiv der Verwandlung von Menschen in Tiere hingewiesen, das Schulz mit Franz Kafka verbindet. Lange Zeit funktionierte daher das Stereotyp „Schulz als polnischer Kafka“.

3 Vgl. die zutreffende Bemerkung von AugSBurger (2008:17): „Die jüdische Herkunft von Schulz ging nicht glatt in eine polnische Identität über.“

4 Es handelt sich um folgende Romane: GRossman, DAVID (1986): ,Ayen ' Erekh: Ahavà (dt.: Stichwort: Liebe); OzICK, CYNTHIA (1987): The Messiah of Stockholm (dt.: Der Messias von Stockholm), RicCARELLI, UGo (1998): Un uomo che forse si chiamava Schulz (dt.: Der Mann, der vielleicht Schulz hieß). 
erster Linie um den Dialog mit philosophischen und anthropologischen Schriften v. a. seiner deutschen Zeitgenossen. SCHMID (2004:119) deutet das Werk von Schulz als ,,wörtliche Umsetzung [...] dreier grundlegender Metaerzählungen der europäischen Moderne": derjenigen von Kant, Freud und Kafka. Diese Bezüge der Schulz'schen interdiskursiven Translation sollen nachfolgend um die Mythos-Idee sowie den psychoanalytischen Diskurs ${ }^{5}$ mit dem Hauptbegriff des ,kollektiven Unbewussten` ergänzt werden.

\subsection{Der Mythos und die mythische Zeit bei Bruno Schulz und Thomas Mann}

Der Mythos wird als Interpretationskategorie auf das Prosawerk von Bruno Schulz auffallend häufig angewendet. In den literarischen, programmatischen und autothematischen Aussagen des Autors aus Drohobycz lassen sich mehrere Bedeutungen dieses Begriffes unterscheiden (vgl. BOLECKI 2006:222f.). Hier werden diejenigen semantischen Varianten berücksichtigt, die das Schaffen von Schulz mit der Reflexion von Thomas Mann und C.G. Jung verbinden, wobei diese Bedeutungen nicht unbedingt scharf voneinander zu trennen sind. In erster Linie wären unter Mythos narrative Bausteine $\mathrm{zu}$ verstehen, die formal oft der antiken Mythologie entnommen werden. Es handelt sich um Stoffe, Motive, Geschichten, Figuren usw., die in abgewandelten Formen in der Literatur immer wieder vorkommen (wie etwa die Verwandlung von Menschen in Tiere oder die Wiederbelebung von Toten), ursprünglich aber dem kollektiven Unbewussten der Menschheit entstammen. Dieser erste Bedeutungsaspekt fällt größtenteils mit der Vorstellung des Jung'schen Archetyps zusammen. Bei Schulz ist der so verstandene Mythos dort anzutreffen, wo der Dichter einen „neuen Mythos“ z. B. aus biblischen Motiven konstruiert (z. B. die „Parabel“ von Anna Csillag in der Erzählung Księga [Das Buch]). Der Mythos kommt auch in Masken oder Kostümen zum Aus-

5 Schulz thematisiert in seinen literarischen und publizistischen Schriften bestimmte Erkenntnisse der Tiefenpsychologie. Es handelt sich vor allem um den Text Aneksja podświadomości [Die Eroberung des Unterbewussten] - die Besprechung des Romans Cudzoziemka [Die Fremde] der polnischen Autorin Maria Kuncewiczowa (SPEINA 1976:29). Obwohl Schulz auf Grund seiner Deutschkenntnisse die Schriften von Freud und Jung im Original einsehen konnte, fehlt bisher ein unmittelbarer Beleg dafür, wann und welche Werke er wirklich gelesen hat - im Unterschied zu seinen Thomas Mann-Lektüren, die recht gut dokumentiert sind. 
druck, die der Erzähler seinen Figuren verleiht (ein Landstreicher als griechischer Gott Pan, der Vater als alttestamentarischer Prophet, das Dienstmädchen Adela als Göttin Pomona) - vgl. JARZĘBSKI (1989:LXXV). GOŚLICKIBAUR (1975) interpretiert die beiden Erzählbände als eine sich kontinuierlich entwickelnde, um den Vater-Sohn-Mythos aufgebaute Geschichte. ${ }^{6}$

Die zweite Auffassung des Mythos hat bei Bruno Schulz einen sprachphilosophischen Charakter. Der Mythos steht für den ursprünglichen Sinn der Sprache, der der Perzeption der Realität und dem ganzen menschlichen Tun zu Grunde liegt. Nach Schulz sei das Wort am Anfang der Geschichte der Menschheit ein mehrdeutiges, metaphorisches Gebilde gewesen, dessen Sinn im Laufe der Zivilisationsentwicklung den Bedürfnissen der alltäglichen Kommunikation angepasst, zunehmend vereinfacht und verflacht wurde. ${ }^{7}$ Diesen Gedanken thematisiert Schulz' wichtigster programmatischer Aufsatz Mityzacja rzeczywistości (Die Mythisierung der Wirklichkeit):

Das Wesen der Wirklichkeit ist der S in n. Was keinen S in n hat, ist für uns nicht wirklich. Jedes Stück Wirklichkeit lebt dadurch, daß es an einem universalen S in n teilhat. Die alten Kosmogonien faßten dies in den Satz: Am Anfang war das Wort. Was unbenannt ist, existiert für uns nicht. Etwas zu benennen bedeutet, es in einen universalen Sinn einzubeziehen. Das isolierte Wort, das Wort als Mosaikstein, ist ein späteres Produkt, es ist bereits ein Resultat der Technik. Das ursprüngliche Wort war ein Phantasiegebilde, das den Sinn der Welt umkreiste, es war ein großes universales Ganzes. (SCHULz 2008:193).

Die Mythisierung der Wirklichkeit wäre demnach die sinnstiftende Tätigkeit des Menschen (des Dichters insbesondere) mit dem Ziel, das Wort auf seinen ursprünglichen, authentischen Sinn zurückzuführen - eine Aufgabe, die mit Mitteln der Poesie erfüllt werden kann und soll.

Die sprachphilosophische Bedeutung des Mythos verweist auf den dritten Aspekt dieses Begriffs, der bei Schulz präsent ist: die ursprüngliche Weltordnung, die es wiederherzustellen gilt, um sich im kosmischen Chaos zurechtzufinden. Unter der „Mythisierung der Welt“" versteht der polnische

6 Obwohl die Autorin den gegennarrativen Charakter der Schulz'schen Erzählungen betont, ergibt ihre Interpretation eine eindeutige Handlung: die Geschichte der Vater-Sohn-Beziehung, die verschiedene Stadien durchläuft - vom Zusammenwirken der beiden über den Bruch, die Flucht des Vaters bis zur Machtergreifung und schließlich wieder der Flucht des Sohnes. OLCHANOwSKI (2001) deutet den Vater zudem als eine archetypische Figur im Sinne von C. G. Jung.

7 Dieser Gedanke erinnert an Ernst Cassirers Auffassung der Sprache - allerdings au rebours. Zu den Ähnlichkeiten und Unterschieden in der sprachphilosophischen Reflexion von Schulz und Cassirer vgl. SZYMAŃSKI (1973:101f.). 
Autor auch den Versuch, die Anarchie des Daseins zu bändigen, die Wirklichkeit ganzheitlich zu erfassen und deren Perzeption auf eine feste Grundlage zu stützen. Dem verunsicherten, im Labyrinth der Welt verlorenen Gegenwartsmenschen sollte mit Hilfe stereotyper Masken eine eindeutige Identität verschafft werden (MILLATI 2006:158). Diese Bemühungen bestehen bei Schulz in der spezifischen Gestaltung der dargestellten Wirklichkeit, wozu in erster Linie die Mythisierung der Zeit gehört. Die geschilderten Ereignisse spielen sich auf zwei Zeitebenen ab: in der historischen Zeit um die Jahrhundertwende, in welche die Kindheit von Bruno Schulz (sowie die des Ich-Erzählers) fällt, und zugleich in einer mythischen, d.h. nicht linearen, zyklischen, umkehrbaren, beliebig oft wiederholbaren Zeit. Diese zweite Zeitebene wird infolge der Dekonstruktion der realen Zeit geschaffen, die der Erzähler durch die kreisförmige Rückkehr zur Vergangenheit, durch die Figur der ,alternativen Zeit“ und der ,zeitlichen Nebengleise“ in Frage stellt. Die erinnerten Vorgänge werden aus der Perspektive eines Kindes erzählt und zugleich vom erwachsenen Erzähler in eine ,zeitlose Immer-Gegenwart“ überführt bzw. rückwirkend als ewige Vergegenwärtigung (DULAIMI 1975:195) interpretiert.

Die Vorstellung der mythischen Zeit lässt den deutschen Rezipienten von Schulz an Thomas Mann denken - mit dem Unterschied, dass der Verfasser der Zimtläden diese Zeit konstruiert, der deutsche Schriftsteller dagegen eher thematisiert. ${ }^{8}$ In seinem Aufsatz Freud und die Zukunft setzt Thomas Mann die Zeit mit einem immer wieder von neuem begangenen Fest in Beziehung: „Ist nicht der Sinn des Festes Wiederkehr als Vergegenwärtigung? [...] Das

8 Vor allem in dem kulturphilosophischen Essay Vorspiel: Höllenfahrt, der den Geschichten Jaakobs vorangeht (1933), des Weiteren in dem Vortrag Freud und die Zukunft (1936) und in dem Selbstkommentar Joseph und seine Brüder. Ein Vortrag (1942), den Bruno Schulz nicht mehr lesen konnte. Eine Mythos-Auffassung, die der Schulz'schen eng verwandt ist, findet sich in Thomas Manns Romanzyklus Joseph und seine Brüder, über den sich Schulz in seinen Briefen mit Begeisterung äußerte. Er konnte natürlich nur diejenigen Teile der Tetralogie lesen, die vor dem Zweiten Weltkrieg erschienen sind. Einen nachhaltigen Eindruck machte auf ihn der Prolog Höllenfahrt - vgl. SCHULZ (1989:422, 446). Allerdings entstanden die Zimtläden und das Sanatorium zur Todesanzeige, noch bevor sich Schulz mit Manns Tetralogie (bzw. mit dessen erstem Teil Die Geschichten Jaakobs) bekannt machen konnte. Aus der Zeit nach der MannLektüre stammt nur der zuvor zitierte Aufsatz Die Mythisierung der Wirklichkeit, dessen Grundgedanken in der Mann'schen Mythos-Idee eine Bestätigung finden. 
Fest ist die Aufhebung der Zeit, ein Vorgang, eine feierliche Handlung, die sich abspielt nach geprägtem Urbild; [...] es gewinnt Gegenwart und kehrt wieder, wie eben Feste wiederkehren in der Zeit [...]“ (MANN 1990:497) eine Reflexion, die später dank Mircea Eliade in die Kulturwissenschaft einging. Diesen Gedanken setzt Schulz in seine Wortkunst um. Er führt die Mythisierung der Zeit - als Element seiner Individualpoetik - zum einen in der Sprache herbei (durch auffällige Rekurrenz von iterativen und unvollendeten Verbformen, die unabgeschlossene, wiederholbare Vorgänge bezeichnen, vgl. STALA 1995:109-111), zum anderen auf der narrativen Ebene, indem alltägliche, banale Gegenstände, Ereignisse und Tätigkeiten durch ständige Wiederkehr und Zusammenstellung u.a. mit vegetativen Zyklen sakrale Würde erhalten und zu einer Zeremonie erhoben werden. So verwandelt sich ein gemeinsames Mittagessen in ein heimliches Ritual, und ein altes Werbeblatt - in das „heilige Buch“. Die Welt der Schulz'schen Prosa wird absichtlich so geformt, dass durch ihre Alltäglichkeit der universale Mythos „durchscheint“"(JARZĘBSKI 2005:129).

Für die Wiederherstellung der ursprünglichen Weltordnung sind zwei Mythen (im Sinne narrativer Bausteine) von Relevanz: der Mythos der Kindheit und derjenige des ,heiligen Buches“. Das Heraufbeschwören der Kindheit bedeutet, dass der Erzähler die spontane Perzeptionsweise eines Kindes annimmt und sie dem Leser aufzwingt - eine Perspektive, die die Dreiteilung der Zeit in Vergangenheit, Gegenwart und Zukunft aufhebt. Die Kindheit des einzelnen Menschen hängt wiederum mit der Kindheit der Menschheit zusammen - einem der Hauptthemen von Thomas Mann, der in seinem Spätwerk nach dem Ursprung des menschlichen Geistes und der menschlichen Gesittung fragt. Der zweite Schulz'sche Grundmythos ist das ,heilige Buch“, das ,unverfälschte Original“. Es symbolisiert die Urquelle jeglichen Sinns, der Erkenntnis, die ursprüngliche Einheit von Geist und Welt, die ewige, kosmische Ordnung, die dem Menschen einleuchtet, sobald er die im „Buch“ verschlüsselten Zeichen entziffert (JARZĘBSKI 1989: LXXVIILXXIX).

Schulz' Auffassung vom Mythos verweist also auf Thomas Mann und über diesen auf die Tiefenpsychologie. Für Mann ist der Mythos ein Kernbegriff der Psychoanalyse, der sich naturgemäß auf die Literatur übertragen lässt: „Das mythische Interesse ist der Psychoanalyse genau so eingeboren, wie allem Dichtertum das psychologische Interesse eingeboren ist." (MANN 1990:493). Der Mythos-Begriff von Mann überschneidet sich vor allem mit der ersten, ,narrativen“ Bedeutung dieser Kategorie bei Schulz. Es handelt 
sich dabei um eine Geschichte, ein überzeitliches Erzählmuster, das in verschiedenen Gestalten im Werdegang der Menschheit immer wieder vorkommt und auf das die Mythologien aller Weltkulturen zurückgehen. Personen, die nach diesem Muster handeln, sind beliebig austauschbar und erfüllen ihre von vornherein vorgeschriebenen Rollen. Die motivische Heterogenität seines Romans Joseph und seine Brüder, der aus biblischen, ägyptischen, altgriechischen u. a. Mythologien schöpft, erklärt Mann damit, dass das Werk

[...] vieles zu vereinigen sucht und, weil es das Menschliche als eine Einheit empfindet und imaginiert, seine Motive, Erinnerungen, Anspielungen, wie seine Sprachlaute aus vielen Sphären borgt. Wie das Jüdisch-Legendäre darin beständig mit anderen, zeitlos behandelten Mythologien unterbaut und dafür durchsichtig gemacht ist, so ist auch sein Titelheld, Joseph, eine durchsichtige und vexatorisch mit der Beleuchtung wechselnde Gestalt: er ist, mit viel Bewußtsein, eine Adonis- und Tammuz-Figur, aber dann gleitet er deutlich in eine Hermes-Rolle [...]. (MANN 1990a:664)

Diese Wechselhaftigkeit der literarischen Figur korrespondiert mit Schulz' Idee der Panmaskerade und der mythischen Masken, die den Protagonisten der Zimtläden zugewiesen werden. Bei Mann ist jeder Mensch zugleich er selbst und ein mythischer Held, der in ausgetretenen Spuren seiner Vorfahren wandelt. Jede individuelle Biographie ist nur eine Wiederholung mythischer Vorbilder, Handlungen und Verhaltensweisen. Dies geht so weit, dass der Mythos die „Legitimation des Lebens“ (MANN 1990:496) überhaupt bildet und den Protagonisten kaum Spielraum übrig lässt, ihre eigenen Biographien frei zu gestalten. Es handelt sich jedesmal um ein „zitathaftes Leben“(MANN 1990:497), eine anthropologische Formel, die die spätere Dekonstruktion vorwegnimmt. Alles, was der Mensch unternimmt, ist nur „Imitation und Nachfolge“ - „eine Lebensauffassung [...], die die Aufgabe des individuellen Daseins darin erblickt, gegebene Formen, ein mythisches Schema, das von den Vätern gegründet wurde, mit Gegenwart auszufüllen und wieder Fleisch werden zu lassen. (MANN 1990b:127). Das „Wandern in ausgetretenen Spuren" betrifft nicht nur das menschliche Leben, sondern auch das Schaffen eines Dichters. Das belegt Thomas Mann selbst, dessen Spätwerk zum größten Teil eine Nacherzählung fremder Stoffe ist. ${ }^{9}$ Das Bewusstsein, dass man mit der Sprache - der alltäglichen genauso wie der poetischen - alte Vorbilder wiederverwertet und ursprüngliche Mythen belebt, kommt auch bei Schulz zum Ausdruck:

Außer den Joseph-Romanen ist es etwa Der Erwählte - eine Paraphrase des Gregorius-Epos Hartmanns von Aue. 
Wenn wir mit umgangssprachlichen Worten operieren, vergessen wir immer, $\mathrm{da} ß$ es Teile vergangener und ewiger Geschichten sind, und daß wir, wie die Barbaren, unsere Häuser aus den Trümmern von Skulpturen und Statuen der Götter errichten. Unsere nüchternsten Begriffe und Definitionen sind entfernte Derivate der Mythen und Geschichten von früher. Selbst das kleinste Körnchen unserer Ideen ist aus der Mythologie hervorgegangen - ist seinerseits verwandelte, verstümmelte, umgeformte Mythologie. Die ursprünglichste Funktion des Geistes ist das Fabulieren, das Erfinden von „Geschichten“. (SCHULZ 2008:184f.)

\subsection{Der Künstler als Medium des kollektiven Unbewussten}

Die bei Bruno Schulz und Thomas Mann vorhandenen Masken und Rollen, die sich zum Mythos im narrativen Sinne zusammenfügen, lassen sich mit Archetypen von C.G. Jung identifizieren - auch wenn beide Dichter einen jeweils anderen Aspekt dieses Konzeptes ins Auge fassen. Mann scheint den Archetyp verhaltenspsychologisch zu verstehen: als „Urnormen“ und „Urformen" der menschlichen Reaktionen und Handlungen in bestimmten existenziellen Situationen. Bei Schulz (der den Terminus übrigens nicht gebraucht) sind die Archetypen mit den vorhin genannten mythischen Erzählbausteinen („Geschichten“), aber auch mit Bildern im visuellen Sinne gleichzusetzen; dies mag mit der Präferenz des Dichters für bildende Künste zusammenhängen. Daher nehmen archetypische Vorstellungen in seiner Erzählung Genialna epoka [Die geniale Epoche] die Gestalt von Zeichnungen an, die dem Unbewussten des kindlichen Ich-Erzählers entspringen.

Die Kindheit und das kollektive Unbewusste sind weitere Motive, die das Schaffen von Bruno Schulz und Thomas Mann über den gemeinsamen Nenner der Jung'schen Psychoanalyse verbinden. In seiner Reflexion geht Mann davon aus, dass das Selbstbewusstsein und die Individualität des vorgeschichtlichen Menschen, sein Gefühl der eigenen Einmaligkeit noch nicht ausgeprägt waren: „Das antike Ich und sein Bewußtsein von sich war ein anderes als das unsere, weniger ausschließlich, weniger scharf umgrenzt. Es stand gleichsam nach hinten offen und nahm vom Gewesenen vieles mit auf, was es gegenwärtig wiederholte, und was mit ihm ,wieder da "war." (MANN 1990:495) Dafür wurde das Leben der vorgeschichtlichen Menschen von ihrer Gruppenzugehörigkeit determiniert: Sie blieben ,mit einem starken Teil ihres Wesens im Mythischen, im Kollektiven befangen“ (MANN 1990a:666) daher ihre Bereitschaft, sich mit einer im Mythos vorgeschriebenen Rolle zu identifizieren. Das Mythische ist für Thomas Mann immer mit dem Kollektiven verbunden; in seinen Joseph-Romanen erzählt er ,,die Geburt des Ich aus 
dem mythischen Kollektiv“ (MANN 1990a:665) - ein deutlicher Nachklang der Überzeugung von Jung, der „natürliche“ Mensch sei ein vollkommen gemeinschaftliches Wesen, dem im Zuge der Abgrenzung der eigenen Person gegen die Umwelt die überwältigende Macht des Kollektiven bewusst wird (vgl. JUNG 2006:84f.). In der Emanzipation des Ich und in der daraus resultierenden anthropozentrischen Weltanschauung sieht Mann die Voraussetzung für den Zivilisationsfortschritt und die Entwicklung der Kultur. Er zieht eine Parallele zwischen der Psychogenese des Kindes und der Menschheitsgeschichte - ein deutlich von der Psychoanalyse inspiriertes Konzept:

Ihr [der Psychoanalyse - K. L.] Zurückdringen in die Kindheit der Einzelseele ist zugleich auch schon das Zurückdringen in die Kindheit des Menschen, ins Primitive und in die Mythik. [...] In der Wortverbindung „Tiefenpsychologie“ hat „Tiefe“ auch zeitlichen Sinn: die Urgründe der Menschenseele sind zugleich auch Urzeit, jene Brunnentiefe der Zeiten, wo der Mythus zu Hause ist und die Urnormen, Urformen des Lebens gründet. Denn Mythus ist Lebensgründung; er ist das zeitlose Schema, die fromme Formel, in die das Leben eingeht, indem es aus dem Unbewußten seine Züge reproduziert. (MANN 1990:493)

Der Diskurs, in dem der Mythos, die Kindheit und das Unbewusste zusammenhängen, steht dem poetologischen Programm von Schulz besonders nahe. In den Erzählbänden Die Zimtläden und Das Sanatorium zur Todesanzeige ist die Überzeugung präsent, dass allen Menschen eine psychische Energie gemeinsam ist, die in der überindividuellen Vorgeschichte der Menschheit wurzelt und in „Bildern“ und „Geschichten“ zum Ausdruck kommt, die sich dem Einzelnen - dem Künstler insbesondere - unwillkürlich aufdrängen. Die einprägsamste Darstellung des kollektiven Unbewussten enthält die Erzählung Wiosna (Der Frühling) im Bild der Wanderung in die „Unterwelt“, „zu den Müttern“:

Wir sind mit unseren Worten am Ende, die schon faseln, irrereden und alles Maß verloren haben. [...] Erst hinter unseren Worten, wo die Macht unserer Magie nicht mehr hinreicht, rauscht dieses dunkle, unfaßbare Element. Das Wort zerfällt dort und löst sich in seine Bestandteile auf, kehrt zu seiner Etymologie zurück, geht wieder in die Tiefe und gleitet in seine dunkle Wurzel hinab. [...] So vollzieht sich in uns ein Rückschritt auf der ganzen Linie, ein Rückzug in die Tiefe, eine Rückkehr in uns selber. So verzweigen wir uns in der Tiefe durch Anamnese [...]. Wir sind auf der anderen Seite, wir sind auf der Sohle der Dinge [...]. Welch ein Kreisen, Verkehr und Gedränge! Was für ein Wimmeln und Wuseln! Wieviel Menschen und Geschlechter, tausendmal vervielfältigte Bibeln und Iliaden! Was für Wanderungen und Tumulte, was für ein Wirrwarr und Durcheinander von Geschichte! Weiter führt dieser Weg nicht mehr. Wir sind auf dem eigentlichen Grund, bei den dunklen Fundamenten, wir sind bei den Müttern! (SCHULZ 1981:157-160) 
Die in der „Unterwelt“, im kollektiven Gedächtnis der Menschheit gespeicherten, ,,aufgeschichteten“ Ideen, Begriffe und Vorstellungen, die sich nicht mehr verbalisieren, sondern nur intuitiv erahnen lassen, sind unschwer mit den Archetypen von Jung zu identifizieren. Auch der Terminus „Anamnese“ ist im Sinne von Jung zu verstehen: als Heraufbefördern von unbewussten Inhalten, ein Zurückgreifen auf archetypische Bilder (vgl. JARZĘBSKI: Fußnote 31 in SCHULZ 1989:158). Diejenigen, die zu diesen Archetypen stets Zugang haben, sind Kinder und Künstler. In beiden offenbart sich das Genie, daher gilt die Kindheit für Schulz als ,geniale Epoche“. Der kindliche Protagonist der gleichnamigen Erzählung ist zugleich auch Zeichner, der seine visionären Bilder nicht sich selbst, sondern einer intuitiven Eingebung, der Stimme seines Unbewussten zuschreibt:

O diese lichtvollen Zeichnungen, die gleichsam unter fremder Hand hervorwuchsen [...]! [...] Von allem Anfang an überkamen mich Zweifel, ob ich tatsächlich ihr [der Zeichnungen - K. L.] Autor sei. Mitunter scheinen sie ein mir selber nicht bewußtes Plagiat zu sein, etwas, was mir eingesagt, zugesteckt, unterschoben wurde... Als ob sich etwas Fremdes für mir unbekannte Zwecke meiner Begeisterung bedient hätte. (SCHULZ 1981:128, 135)

Der Künstler unterscheidet sich vom Kind dadurch, dass er in der Lage ist, über seine Rolle als Medium des kollektiven Unbewussten zu reflektieren: „Woher nähmen die Schreiber ihre Stoffe, woher sonst schöpften sie den Mut zum Fabulieren, spürten sie nicht diese Reserven, diese Kapitale, diese hundertfachen Mannigfaltigkeiten hinter sich, von denen die Unterwelt vibriert.“ (SCHULZ 1981:160) ${ }^{10}$ Das kollektive Unbewusste ist das, was der Kreativität des Dichters Rückhalt bietet. Diese Überzeugung korrespondiert mit Jungs Idee, dass in jedem genialen, visionären Dichterwerk ein Bild des kollektiven Unbewussten durchscheint. Die spezifisch künstlerische Psychologie sei „eine kollektive und keine persönliche Angelegenheit“ und der Künstler - ein „,Kollektivmensch, ein Träger und Gestalter der unbewußt tätigen Seele der Menschheit.“(JUNG 1990:142). Genauso begreift auch Bruno Schulz die Aufgabe des Dichters: Er soll Mythen beleben, das Wort auf seinen ursprünglichen Sinn zurückführen, die im kollektiven Unbewussten verwurzelten Archetypen zu Tage fördern und ihnen poetische Gestalt verleihen.

10 Der Begriff ,Schreiber“ ist an dieser Stelle vielmehr als ,Dichter` zu verstehen, was auch die adäquate Übersetzung des Schulz'schen ,,pisarze“ wäre (im polnischen Original: „Skądże by pisarze brali swe koncepcje [...]“-SchUlz 1989:160). 


\section{Die künstlerische Verarbeitung von Archetypen als Aufgabe des Dichters: Bruno Schulz in deutschen Übersetzungen}

Die Umsetzung des psychoanalytischen Diskurses im Schaffen von Bruno Schulz sowie die Parallelen zu Thomas Manns Mythos-Konzept dürften den deutschen Lesern nicht entgangen sein. Man kann die Behauptung wagen, dass diese Affinität die intensive Rezeption der Prosa des polnischen Autors, die im deutschen Sprachraum Anfang der 1960er Jahre einsetzte, mit herbeiführte. Eine andere Frage bleibt, inwiefern die vorhandenen Übersetzungen diesen interdiskursiven Translationsakt von Schulz widerspiegeln. Das Gesamtwerk des polnischen Autors liegt heute in der deutschen Fassung von Josef Hahn aus den 1960er Jahren vor, die immer wieder neu aufgelegt wird und daher als gewissermaßen kanonisch gilt. ${ }^{11}$ Erst 2008 erschien die Neuübersetzung von Doreen Daume, die den Band Die Zimtläden und den Essay Die Mythisierung der Wirklichkeit umfasst. Nachstehend werden Passagen aus der letzteren Schrift ihren beiden Übersetzungen gegenübergestellt, außerdem wird eine Stelle aus der Erzählung Wiosna (Der Frühling) mit der einzig verfügbaren Version von Josef Hahn verglichen. In den beiden Originaltexten - dem literarischen und dem metapoetischen - schwingen die Ideen des kollektiven Unbewussten, der Mythen und Archetypen besonders deutlich mit. Beiden ist gemeinsam, dass sie die Aufgabe des Dichters thematisieren, also den Diskurs der Tiefenpsychologie auf das literarische Gebiet übertragen.

In Die Mythisierung der Wirklichkeit knüpft Schulz an den verlorengegangenen, authentischen Sinn der Sprache - den Mythos in sprachphilosophischer Bedeutung - an:

Poezja odpoznaje te sensy stracone, przywraca słowom ich miejsce, łączy je według dawnych znaczeń. U poety słowo opamiętuje się niejako na swój sens istotny, rozkwita i rozwija się spontanicznie według praw własnych, odzyskuje swą integralność. Dlatego wszelka poezja jest mitologizowaniem, dąży do odtworzenia mitów o świecie. (SCHULz 1989:366f.)

Die Poesie erkennt diesen verlorenen Sinn, gibt den Wörtern ihren Platz wieder, verbindet sie gemäß ihren alten Bedeutungen. Beim Dichter erinnert sich das Wort gewissermaßen an seinen wirklichen Sinn, blüht auf, entfaltet sich spontan nach den eigenen Gesetzen und gewinnt seine Integrität wieder. Deshalb ist

11 Sie bildet auch die Grundlage für die deutsche Schulz-Forschung sowie für intersemiotische Transpositionen seiner Prosa (z.B. Kataloge zu Ausstellungen seiner Graphiken). Mehrere publizistische Texte wurden auch von Mikołaj Dutsch übersetzt (z.B. in SCHULZ 1992). 
jegliche Poesie Mythologisieren und sehnt sich nach der Wiedergabe der Mythen der Welt. (SCHULZ 1992:241; Übers. Josef Hahn)

Die Poesie erkennt diesen verlorenen Sinn wieder, sie gibt den Worten ihren Ort zurück und fügt sie gemäß ihren früheren Bedeutungen wieder zusammen. Bei einem Dichter besinnt sich das Wort gleichsam auf seinen wesentlichen Sinn, es blüht auf und entwickelt sich spontan nach eigenen Gesetzen, es erlangt seine Integralität zurück. Deshalb ist jede Poesie Mythologisierung, sie trachtet danach, die Welt-Mythen wiederherzustellen. (SCHULz 2008:195; Übers. Doreen Daume)

Die Rekonstruktion der Mythen der Welt - denn so ist das polnische Nominalverb ,odtworzenie' zu verstehen - bedeutet im Kontext der Schulz'schen Sprachphilosophie die Vermittlung von Archetypen, die - selbst unaussprechbar - der Übersetzung in einen Mythos, in ein Symbol bedürfen. Der Dichter leistet hier eine kreative Arbeit, indem er für den Archetyp - eine „noch unmittelbare, seelische Gegebenheit“ (JUNG 1957:13) - nach dessen Bewusstwerdung und Wahrnehmung einen passenden Mythos findet. Diese aktive Tätigkeit des Dichters geht aus der Übersetzung Daumes hervor: Das Wiederherstellen der „Welt-Mythen“ legt einen mühsamen Aufbauprozess nahe, bei dem der Dichter seinen Beitrag zu einem womöglich neuen poetischen Gebilde leistet. Die Fassung von Hahn dagegen, die von der „Wiedergabe der Mythen der Welt" spricht, vermittelt das Bild eines passiven Nacherzählers: Der Dichter soll bereits vorhandene Mythen lediglich einsammeln und treu wiedergeben. Der Begriff ,Mythos ‘ verliert in Hahns Übersetzung seinen psychoanalytischen Anklang und verweist eher auf die Mythologie als Sammlung von Geschichten über Götter und Helden. Aus der deutschen Version von Hahn ergibt sich somit ein imitativer Charakter der Poesie, wohingegen Daume deren schöpferischen Sinn unterstreicht und dadurch dem polnischen Original wesentlich näher steht. Die Übertragung Hahns scheint die Schulz'sche Affinität zu Jungs Idee der Archetypen auch an anderen Stellen eher zu verdunkeln als hervorzuheben. Schulz spricht mehrmals von dem Prozess der Sinngebung durch das poetische Wort, infolgedessen eine für den Menschen wahrnehmbare, begreifliche Wirklichkeit entsteht:

Duch ludzki niestrudzony jest w glosowaniu życia przy pomocy mitów, w „usensowianiu” rzeczywistości. [...] Proces usensowiania świata jest ściśle związany ze słowem. (SCHULZ 1989:367)

An dieser Stelle gibt die Übertragung von Daume die nominalisierte Neuschöpfung, usensowianie ' adäquat wieder:

Der menschliche Geist ist unermüdlich, das Leben durch Mythen zu glossieren, die Wirklichkeit mit Sinn zu versehen. [...] Die Welt mit Sinn zu versehen ist ein Prozess, der eng mit dem Wort verbunden ist. (SCHULZ 2008:196) 
Die Version von Hahn dagegen hat einen sensualistischen Anklang: Das Wort des Dichters soll die Welt sinnlich wahrnehmbar machen:

Der unermüdliche menschliche Geist besteht im Glossieren des Lebens unter Zuhilfenahme der Mythen und in der „Versinnlichung“ der Wirklichkeit. [...] Der Prozeß der Versinnlichung der Welt ist aufs engste mit dem Wort verbunden. (SCHULZ 1992:242)

Diese Passage impliziert eine ganz andere Sprachphilosophie als diejenige von Schulz. Im Original besteht die Sinngebung darin, dass der Mensch der unendlichen, unbenannten Materie (sprachliche) Formen gibt. Der Welt Sinn zu verleihen, kann man aber auch dahingehend verstehen, dass der Dichter nach passenden Symbolen ${ }^{12}$ für das Unausgesprochene sucht, die ihm helfen, das Chaos des kollektiven Unbewussten mit dessen vage erahnten Archetypen in eine für ihn verständliche Ordnung zu bringen. Die symbolische Darstellung bedeutet also eine Bewusstseinsleistung und nicht unbedingt sinnliche Wahrnehmbarkeit, wie es die Formulierung von Hahn, die „Versinnlichung“ der Welt, nahe legt. Aus der Übersetzung geht hervor, dass der Dichter die Inhalte des Unbewussten in anschaulichen, womöglich visuellen Formen festhält. Diese Auslegung blendet den psychoanalytischen Kontext der Schulz'schen Reflexion aus, da die Archetypen nach Jung weder beschreibbar noch darstellbar sind (vgl. JuNG 1957:13, Fußnote 1). Andererseits stellt der Übersetzer einen intertextuellen Bezug her zwischen den theoretischen Überlegungen des Dichters und dem Motiv der visionären Zeichnungen des Kindes in der Erzählung Die geniale Epoche.

Es gibt allerdings auch Anlass zu vermuten, dass der Übersetzer die Inspirationen von Jung in den Erzählungen des polnischen Autors erkannte. Es handelt sich um den Gebrauch der Adjektive bzw. Adverbien bewusst unbewusst in der Übersetzung, wo zuweilen ein deutlich Jung' scher Sinn mitschwingt. In dem bereits angeführten Satz aus der Erzählung Die geniale Epoche bezeichnet der Erzähler seine eingebungsvollen Bilder als „mimowolny plagiat“ - ein ,unwillkürliches Plagiat“, was Hahn als „ein mir selber nicht bewußtes Plagiat“ übersetzt - eine womöglich intendierte Anspielung auf die in der Psychoanalyse verwurzelte Gegenüberstellung zwischen Bewusstem und Unbewusstem. Versteckte Hinweise auf die Übertragung des psychoanalytischen Diskurses in die Schulz'sche Prosa finden sich vor allem in

12 Hier ist die Jung'sche Unterscheidung zwischen Symbol und Allegorie von Bedeutung: Während die Allegorie einen bewussten Inhalt paraphrasiert, sei das Symbol „ein bestmöglicher Ausdruck für einen erst geahnten, aber noch unerkannten unbewußten Inhalt.“ (Jung 1957:14, Fußnote 1) 
der Übersetzung der Erzählung Wiosna (Der Frühling), die bisher nur von Josef Hahn übertragen wurde. Nachstehend folgt eine Passage, in der Schulz den Abstieg ins kollektive Unbewusste poetisch darstellt - eine Schilderung, die an den unergründlichen „Brunnen der Vergangenheit“ bei Thomas Mann anknüpft:

Teraz wreszcie rozumie się ten wielki i smutny mechanizm wiosny. Ach, ona rośnie na historiach. Ile zdarzeń, ile dziejów, ile losów! Wszystko cośmy kiedykolwiek czytali, wszystkie zasłyszane historie i wszystkie te, które się nam majaczą od dzieciństwa - nigdy nie zasłyszane - tu, nie gdzie indziej jest ich dom i ojczyzna. [...] Idziesz z przymkniętymi oczyma w tym cieple szeptów, uśmiechów i propozycyj, nagabywany bez końca, nakłuwany tysiąckrotnie pytaniami, jak milionami słodkich ssawek komarzych. Chciałyby, byś wziął coś od nich, cokolwiek, szczyptę choć tych bezcielesnych, szepcących dziejów, i przyjął to w swe młode życie, w krew swoją, i ocalił i żył z tym dalej. Bo czymże jest wiosna, jeśli nie zmartwychwstaniem historyj. (ScHUlz 1989:160f.)

Jetzt endlich wird der große und traurige Mechanismus des Frühlings verständlich. Ach, er wächst auf Geschichte. So viele Erfolge, so viele Handlungen, so viele Schicksale! Alles, was wir jemals gelesen, alle vernommenen Geschichten und alle niemals vernommenen, die uns von Kindheit an als Träume verfolgen, sind hier: nirgends anders ist ihr Haus und ihr Vaterland. [...] Du gehst mit geschlossenen Augen in dieser flüsternden, lächelnden, verheißungsvollen Wärme dahin, unendlich belästigt, tausendfältig von Fragen wie von Millionen süßer Mückenstiche pikiert. Ich wollte, du könntest etwas mitnehmen davon, irgend etwas, wenigstens eine Fingerspitze voll dieser körperlosen, flüsternden Ereignisse, und sie in dein junges Leben aufnehmen, in dein Blut, und gerettet werden und damit weiterleben. Worin soll der Frühling sonst bestehen, wenn nicht in der Auferstehung der Geschichte von den Toten! (SCHULz 1981:160)

Der Frühling lässt sich an dieser Stelle mit Poesie identifizieren, die aus den im kollektiven Unbewussten gespeicherten „Geschichten“ schöpft - sowohl aus denen, die man in der Kindheit z.B. als Märchen erzählt bekommt, als auch denen, die dem Menschen bloß undeutlich, wie Trugbilder oder Fieberphantasien erscheinen -, denn das bedeutet das Verb majaczyć. Hahn spricht an dieser Stelle von Geschichten, „die uns von Kindheit an als Träume verfolgen“. Der Traum als Schlüsselbegriff der Psychoanalyse kommt bei Schulz explizit nicht vor, wird aber in die Übersetzung hineininterpretiert. Auch ansonsten ist die deutsche Version des angeführten Abschnitts in mancher Hinsicht verfehlt. Schulz gebraucht mehrmals den Plural „historie“ ,Geschichten', abwechselnd mit dem gleichbedeutenden Kollektivum dzieje. Es dürfte ein Echo der mythischen „Geschichten“ von Thomas Mann sein, die in den Joseph-Romanen erzählt werden (vgl. etwa den Titel Die Geschichten Jaakobs). Für Schulz bedeuten „historie“ den - meist nicht bewusst 
gewordenen, gleichsam in Archetypen schlummernden - Stoff der Dichtung. Den Satz, dass der Frühling , auf Geschichten wächst“ (,ona rośnie na historiach"), kann man so verstehen, dass die Poesie sich aus Archetypen speist. Der Übersetzer erkennt die Bedeutung der Mehrzahl „historie“ offensichtlich nicht und führt eine im Original nicht motivierte Differenzierung zwischen ,Geschichte' und ,Geschichten ' ein. Die ,Geschichte', auf der der Frühling wächst, ließe sich als eine Folge historischer Ereignisse interpretieren, was zu der Missdeutung führt, Poesie solle den politischen und gesellschaftlichen Werdegang der Menschheit zum Inhalt haben. Eine ähnlich irreführende und die Aussage von Schulz entstellende Auslegung impliziert der Satz, dass der Frühling ,in der Auferstehung der Geschichte von den Toten“ besteht. Dass der Sammelbegriff dzieje synonym mit historie verwendet wird, scheint dem Übersetzer ebenfalls zu entgehen, da er das erste Substantiv als „Handlungen“ oder „Ereignisse“ wiedergibt. ${ }^{13}$ Die beiden deutschen Wörter haben einen konkreten Charakter und verankern das Erzählte fest in der Realität, in der Gegenwart, während das polnische Kollektivum dzieje sich auf die Vergangenheit bezieht und das Element der Rückbesinnung enthält. Daher führt der deutsche Text eine unnötige Differenzierung der Begriffe dort ein, wo es angebracht wäre, das Signalwort historie bzw. dzieje (als ,Geschichten') konsequent beizubehalten und lässt den Zielleser den Bezug der Schulz'schen Vorstellungen zu Jungs Archetypen nicht nachvollziehen.

Was die Erzählung Der Frühling mit dem Essay Die Mythisierung der Wirklichkeit verbindet, ist die Vermittlung der Aufgabe des Dichters. In dem angeführten polnischen Text wird der Angesprochene - den man mit dem Dichter bzw. Künstler überhaupt identifizieren darf - aufgefordert, die „Geschichten“ aus der „Unterwelt“ des kollektiven Unbewussten hervorzuholen, „in sein Leben aufzunehmen“ (d.h. zu verinnerlichen und zu reflektieren) und durch künstlerische Verarbeitung zu retten. Dieses Programm wird in der Übersetzung nicht wiedergegeben. In der Fassung von Hahn kommt es zu einer im Ausgangstext nicht vorhandenen Zersplitterung der erzählenden Instanz in ein „Ich“ und ein „Du“ (,Ich wollte, du könntest etwas nehmen davon $[\ldots]$ “ - im Original sind es die „Geschichten“, die sich direkt an das Du wenden). Außerdem ergibt sich aus der syntaktischen Satzkonstruktion der Übersetzung, dass es der Dichter ist, der durch die Internalisierung der „Ereignisse“ gerettet werden soll: „Ich wollte, du könntest etwas mitnehmen

13 Genauso wie an anderen Stellen in der Erzählung Tyle jest nie urodzonych dziejów (SCHULZ 1989:161) - „Es gibt so viele ungeborene Ereignisse“ (SCHULZ 1981:161). 
davon, irgend etwas, wenigstens eine Fingerspitze voll dieser körperlosen, flüsternden Ereignisse, und sie in dein junges Leben aufnehmen, in dein Blut, und gerettet werden und damit weiterleben." Es sind also nicht die Archetypen, die durch Versprachlichung oder sonstige mythische Formgebung in die Sphäre der Kunst übertragen und damit gerettet werden, sondern der Dichter ist auf seine kreative Tätigkeit angewiesen, von der sein Weiterleben und seine Selbstbehauptung abhängen. Diese inadäquate, irreführende Verdeutschung ist folgenschwer für das Bild der Schulz'schen Prosa, das dem deutschen Leser präsentiert wird. Die Rettung durch Kunst, Poesie als Heilmittel für den Dichter hat mit der kunstphilosophischen Reflexion des polnischen Autors wenig gemeinsam. Die Kontexte, die für das Werk von Schulz ausschlaggebend sind, d.h. der in die Literatur umgesetzte psychoanalytische Diskurs, werden in der deutschen Fassung nicht bzw. nur unzureichend aktiviert.

\section{Schlussbemerkung}

Ulrich Schmid weist zu Recht auf den psychoanalytischen Diskurs von Freud als eine der großen modernen Metaerzählungen hin, die Schulz in seine Prosa umsetzt. Er vertritt die These, Schulz distanziere sich von der Möglichkeit der Psychoanalyse als gültiger Interpretationsmethode, indem er die Voraussetzung der Psychogenese - dass jeder Mensch bestimmte Stationen einer typischen Biographie durchlaufen muss - durch das Außergewöhnliche, Skurrile am Verhalten seiner Helden in Frage stellt (SCHMID 2004:126f.). Dem ist entgegenzuhalten, dass die mythischen Rollen und Masken, die den Protagonisten zugewiesen werden, sich auf die Gesamtheit der überindividuellen Erfahrungen beziehen - Archetypen, die allen Menschen gemeinsam sind. Wenn also Schulz den psychoanalytischen Diskurs in der Freud'schen Ausprägung ablehnt, so ist dessen Weiterentwicklung durch Jung für den Verfasser der Zimtläden eine positive Orientierungsgröße.

Die komparatistische Analyse legt zwei Schlussfolgerungen nahe. Erstens: Der Begriff Übersetzer bzw. Translator sollte in der Literaturwissenschaft nach dem ,translational turn' seinen Geltungsbereich erweitern. Ein Autor, der einen fremdsprachigen geisteswissenschaftlichen Diskurs aufgreift und in seine literarischen Werke umsetzt, vollzieht damit einen Translationsakt; er wird selbst zum Übersetzer, und die Leitbegriffe des Diskurses sind seine translatorischen Bezugsgrößen. Zweitens: Auch wenn der Verfasser einer herkömmlichen interlingualen Übersetzung den im Ausgangstext verarbeite- 
ten Diskurs erkennt und richtig verortet, so bedeutet das noch lange nicht, dass er in der Lage ist, die ursprünglichen Bezugsgrößen des Originalautors im Zieltext zu rekonstruieren. Im Gegenteil: Gerade diese - wie der Mythos und der Archetyp bei Schulz - bereiten dem Übersetzer die meisten Schwierigkeiten! Hinter jedem Begriff (auch wenn er, wie der Archetyp, im Ausgangstext nur implizit vorhanden ist) verbirgt sich ein philosophisches Konzept, das nicht immer konsequent gedacht und in sich stimmig ist, das der Übersetzer aber nachvollziehen sollte. Die Analyse hat gezeigt, dass es einem Übersetzer der Texte von Bruno Schulz nicht immer leichtfällt, den Zielrezipienten zu überzeugen, dass in dieser Prosa eine große europäische Metaerzählung realisiert wird.

\section{Literatur}

Augsburger, Janis (2008): Masochismen. Mythologisierung als Krisen-Ästhetik bei Bruno Schulz. Hannover.

BACHMANN-MEDICK, DORIS (1996 / 2004$):$ Multikultur oder kulturelle Differenzen? Neue Konzepte von Weltliteratur und Übersetzung in postkolonialer Perspektive. In: BachmanN-Medick, Doris (ed.): Kultur als Text. Tübingen/Basel, 262-296.

- (2004a): Kulturanthropologie und Übersetzung. In: KITTEL, HARALD et al. (eds.): Übersetzung - Translation - Traduction. Ein internationales Handbuch zur Übersetzungsforschung. 1. Teilband. Berlin/New York, 155-165.

- (2006 / $\left.{ }^{3} 2009\right)$ : Cultural Turns. Neuorientierungen in den Kulturwissenschaften. Reinbek bei Hamburg.

Bolecki, WŁodZiMierz (2006): Mit. [Mythos]. In: Bolecki / JARZĘBSKI / RosieK, $222 \mathrm{f}$.

BOLECKI, WŁODZIMIERZ / JARZĘBSKI, JERZy / ROSIEK, STANISŁAW (eds.) (2006): Stownik schulzowski. [Schulz-Wörterbuch]. Gdańsk.

BRzostowsKA-TERESZKIEWICZ, TAMARA (2004): Komparatystyka literacka wobec translatologii. Przeglad stanowisk badawczych. [Vergleichende Literaturwissenschaft und Translationswissenschaft. Übersicht über die Forschungsstandpunkte]. In: Przestrzenie Teorii 3/4:279-321.

Dulaimi, KARIN (1975): Der Mythosbegriff im Werk von Bruno Schulz. München.

GoŚLicki-BAur, Elisabeth (1975): Die Prosa von Bruno Schulz. Bern/Frankfurt (M.).

Grossman, DAvid (1986): ,Ayen' Erekh: Ahavà. Jerusalem.

- (1991): Stichwort: Liebe. Aus dem Hebräischen von Judith Brüll. München/Wien.

JARZĘBSKI, JERZY (1989): Wstęp. [Einführung]. In: SCHUlZ, III-CXXV.

- (2005): Prowincja Centrum. Przypisy do Schulza. [Provinz - Zentrum. Anmerkungen zu Bruno Schulz ]. Kraków. 
Jung, CARL Gustav (1957): Über die Archetypen des kollektiven Unbewussten. In: Jung, Carl Gustav: Bewusstes und Unbewusstes. Beiträge zur Psychologie. Mit einem Vorwort von Prof. Dr. E. Böhler und einem Nachwort von Aniela Jaffé. Frankfurt (M.)/Hamburg, 11-53.

- (1985 / ${ }^{3}$ 1990): Psychologie und Dichtung. In: BARZ, Helmut et al. (eds.): Grundwerk C.G. Jung. In neun Bänden. Bd. 9: Mensch und Kultur. Olten/Freiburg im Breisgau, 124-146.

- (1995 / 2006): Über Schillers Ideen zum Typenproblem. In: JUNG, CARL GuSTAV: Psychologische Typen. Düsseldorf, 68-141.

ManN, Thomas (1990): Freud und die Zukunft. In: Reden und Aufsätze (1). Gesammelte Werke in dreizehn Bänden. Bd. IX. Frankfurt (M.), 478-501.

- (1990a): Joseph und seine Brüder. Ein Vortrag. In: Reden und Aufsätze (3). Gesammelte Werke in dreizehn Bänden. Bd. XI. Frankfurt (M.), 654-669.

- (1990b): Joseph und seine Brüder. Gesammelte Werke in dreizehn Bänden. Bd. IV. Frankfurt (M.).

Millati, PIOTR (2006): Ironia. [Ironie]. In: BOlECKI / JARZĘBSKI / RosieK, 157-159.

OlCHANOWSKI, TOMASZ (2001): Jungowska interpretacja mitu ojca w prozie Brunona Schulza. [Die Interpretation des Vater-Mythos in der Prosa von Bruno Schulz unter Anlehnung an C. G. Jung]. Białystok.

OzICK, CYNTHIA (1987): The Messiah of Stockholm. New York.

- (1990): Der Messias von Stockholm. Aus dem Amerikanischen von Melanie Walz. München/Zürich.

RiCCARELLI, UGO (1998): Un uomo che forse si chiamava Schulz. Casale Monferrato.

- (1999): Der Mann, der vielleicht Schulz hieß. Aus dem Italienischen von Sylvia Höfer. München.

SCHMID, UlRICH (2004): Die Realisierung der modernen Metaerzählungen bei Bruno Schulz (Kant, Freud, Kafka). In: Hodel, Robert (ed.): Zentrum und Peripherie in den slavischen und baltischen Sprachen und Literaturen. Bern (=Slavica Helvetica 71), 119-134.

Schulz, BRUNo (1933 / 1989): Opowiadania, wybór esejów i listów. [Erzählungen, Auswahl von Essays und Briefen]. Wrocław/Warszawa/Kraków/Gdańsk/Łódź.

- (1981): Die Zimtläden und alle anderen Erzählungen. Aus dem Polnischen übersetzt von Josef Hahn. Frankfurt (M.).

- (1992): Die Wirklichkeit ist Schatten des Wortes. Aufsätze und Briefe. Hrsg. von Jerzy Ficowski. Aus dem Polnischen von Mikołaj Dutsch und Josef Hahn. München.

- (2008): Die Mythisierung der Wirklichkeit. In: Schulz, Bruno: Die Zimtläden. Aus dem Polnischen von Doreen Daume. München, 193-197.

SPEINA, JERZY (1976): Bruno Schulz wobec psychoanalizy. [Bruno Schulz und die Psychoanalyse]. In: Czaplowa, KAZIMIERA (ed.): Studia o prozie Brunona Schulza. Katowice, 17-30. 
Katarzyna Lukas

STALA, KRZYSZTOF (1995): Na marginesach rzeczywistości. O paradoksach przedstawiania w twórczości Brunona Schulza. [Am Rande der Wirklichkeit. Zu den Paradoxen der Darstellung im Werk von Bruno Schulz]. Warszawa.

SZYMAŃSKI, WIESŁAW PAWEŁ (1973): Na poczq̨tku byto stowo. O twórczości Brunona Schulza. [Am Anfang war das Wort. Über das Werk von Bruno Schulz]. In: SZYMAŃSKI, WIESŁAW PAWEŁ: Outsiderzy i stowiarze. Eseje, szkice, interpretacje. [Außenseiter und Wortschaffende. Essays, Skizzen, Interpretationen]. Wrocław/Warszawa/ Kraków/Gdańsk, 95-128.

Toro, Alfonso De (2003): Jenseits von Postmoderne und Postkolonialität. Materialien zu einem Modell der Hybridität und des Körpers als transrelationalen, transversalen und transmedialen Wissenschaftskonzept. In: HAMANN, CHRISTOPH / SIEBER, CORNElia (eds.): Räume der Hybridität. Zur Aktualität postkolonialer Konzepte. Hildesheim/Zürich/New York, 15-52. 